\title{
FLORA OF KIOWA COUNTY, OKLAHOMA
}

\author{
Master's Thesis \\ Oklahoma Agricultural and Mechanical College \\ [Oklahoma State University] \\ 1938
}

Lottie Opal Baldock

Keywords: distribution, ecology, historic, vascular

\begin{abstract}
[ABSTRACT]
This paper presents the results of taxonomic and ecological studies of the plants of Kiowa County, Oklahoma. The collections were begun in 1933 and continued until the summer of 1938; however, little intensive collecting was done until the spring and summer of 1938. The Flora of Kiowa County, Oklahoma includes six species of pteridophytes, one species of gymnosperms, and 489 species of angiosperms. More than one third of these are in Compositae, Gramineae, and Leguminosae. There are 81 families represented. The 11 largest families, with the number of species are Compositae, 86; Gramineae, 58; Leguminosae, 41; Onagraceae, 17; Euphorbiaceae, 16; Cruciferae, 16; Polygonaceae, and Solanaceae, 12 each; Asclepiadaceae, Cyperaceae, and Labiatae, 11 each. The three largest families comprise 37.4 per cent of the total number of species.
\end{abstract}

[Species names used in the original thesis which appear in brackets have been updated using the USDA Plants Database.]

\section{PREFACE}

The main value of studies such as this is to establish the distribution of species and to observe the varying ecological conditions in which the plants under consideration are growing.

Plants considered in this study and included in the list are native wild species and mainly indigenous to the county; however, a few species of cultivated plants are listed in cases where they have escaped cultivation and seem to have established themselves in the new habitat.

The author does not aim to give a complete list of the vascular plants of the county as the time was limited, and such a survey is a fit subject for more advanced graduate work.

\section{INTRODUCTION}

This paper presents the results of taxonomic and ecological studies of the plants of Kiowa County, Oklahoma (Figure). The collections were begun in 1933 and continued until the summer of 1938; however, little intensive collecting was done until the spring and summer of 1938. More systematic work has been done with the spring and summer flowering plants than with those flowering in the fall. In most cases the nomenclature is that of Gray's Manual (Robinson and Fernald 1908); however, the Oklahoma Flora by Stemen and Meyers (1937) was used as a check, and in some cases plants were listed in the latter publication only. For the grasses, Hitchcock (1935) was the final authority. 


\section{LOCATION AND SIZE}

Kiowa County is in southwestern Oklahoma. It comprises an area of 1,025 square miles, or 656,000 acres $\left[2,655 \mathrm{~km}^{2}\right]$. The county is bounded by Washita on the north, Greer and Jackson on the west, Tillman on the south, and Comanche and Caddo counties on the east. Hobart, the county seat and largest town, is located in the northwestern part, about 136 miles [219 $\mathrm{km}$ ] southwest of Oklahoma City. The elevation at Hobart is 1,536 feet [468 m] (Wahlgren).

\section{HISTORY}

In 1834, a large military expedition was sent out from Forts Gibson and Towson to stop the warfare among the Indians and to pay a visit to the wild bands of Kiowas, Wichitas, and Comanches who lived among the Wichita Mountains. This was probably the first official expedition to reach any part of Kiowa County. They explored the region about the Wichita Mountains going as far west as North Fork Red River. George Catlin, the famous painter of Indian pictures, was with this expedition and left many pictures of things he observed.

The county was opened to settlement by a proclamation of President McKinley on July 4, 1901. The land was allotted by drawing for a choice. This county was formerly a part of the Kiowa, Comanche, and Apache Indian Reservation. In 1910, a part of the county was taken with a part of Comanche County to form Swanson County (Goke and Holopeter 1931), but the creation of Swanson County was declared illegal by a decision of the Supreme Court of Oklahoma, August 9, 1911, and the territory was restored to the former counties. A part was annexed to Tillman County.

The main industry is farming, with cotton and wheat as the leading crops.
Quarrying of granite is carried on to a small extent.

Original dominant vegetation in the county consisted of grasses, a scattered growth of mesquite (Prosopis glandulosa Torr. var. glandulosa [=Prosopis juliflora glandulosa]), and cactus (Opuntia bumifusa (Raf.) Raf.). Along the streams, cottonwood (Populus deltoids W. Bartram ex Marshall), elm (Ulmus americana L.), and pecan (Carya illinoiensis (Wagenh.) K. Koch) were in predominance. During the winter, the grasses in the valley provide the chief source of feed for livestock. Before the land was open for settlement, these valleys were highly prized among the Indians for grazing purposes.

\section{TOPOGRAPHY}

The Wichita Mountains in the south central and eastern portion rise abruptly above the gently rolling plains which are characteristic of the prairies. The mountains are composed of igneous rocks surrounded by sedimentary formations. The igneous rocks are pre-cambrian, but younger than the Proterozoic rocks which they have intruded. Most of the rock is medium to fine grained pink granite, except those of the northern range in the eastern part of the county which are made up of limestone. The granite mountains are covered with a scrubby growth of oaks, but the limestone hills are comparatively barren. The southward facing escarpment which crosses the northern part of the county shows a distinctly different physical feature. This escarpment is composed of calcareous ledges of the Blaine formation (Sawyer 1929).

\section{STREAMS AND DRAINAGE}

North Fork Red River, bounding the county on the west, and Washita River, along the northeastern edge, are the two largest streams. Most of the drainage waters flow through them from their several 
tributaries. East Fork of Deep Red Creek and its tributaries with East, West, and Middle Otter Creeks drain the lower southern section. North Fork Red River with Elk Creek and their tributaries drain the western section. The northeastern section of the county is drained by Washita River and Rainy Mountain, Saddle Mountain, and Stinking Creeks.

\section{SOIL}

The soils of 95 per cent of the area of Kiowa County are heavy in texture either in the surface soil or subsoil, with clay loam mainly in both; the rest, which occur in irregularly shaped areas in different parts of the county, are sandy. The sandy soils are found along the two rivers mentioned, at the western boundary and the northeastern corner. The outstanding difference between the sandy soils and the clay loam soils is that the material of the sandy areas is much more friable throughout the surface soil and subsoil, continuing to a depth of several inches.

Foard silt loam comprises about 128,896 acres [522 km²] (Goke and Holopeter 1931) or 19.6 per cent of the total. This type has a dark-brown surface soil that extends to a depth of six inches, where it gradually passes downward to a dark-brown or brown heavy plastic subsoil. The color in this layer gradually changes to a yellowish-brown. At a depth of 18 inches [ $46 \mathrm{~cm}$ ], lime is present in sufficient quantitites to effervesce in acid.

Tillman clay loam is next in importance with 112,064 acres [454 $\mathrm{km}^{2}$ ] or 17.1 per cent. It has a chocolate-brown friable surface soil that passes at a depth of six inches $[15 \mathrm{~cm}]$ into a dark-brown friable subsurface soil. At about 12 inches [30 cm], this changes to a chocolate-brown or reddish-brown clay subsoil which is tough and plastic when wet and very hard and dense when dry. At a depth of about 24 inches $[60 \mathrm{~cm}]$, lime is first reached in the form of hard concretions or in a finely disseminated form.

The third important type of soil is Vernon clay loam which covers 65,536 acres [265 $\mathrm{km}^{2}$ ] or 10.0 per cent of the land. The surface soil of Vernon clay loam consists of reddish-brown, brown, or chocolate-brown friable material to a depth of four inches. The subsoil is reddish-brown granular clay loam which continues to a depth of about 12 inches [30 cm] where it changes into a reddish-brown clay which is plastic when wet but very hard when dry. This soil is found chiefly on slopes along the valleys and in areas that are cut by many drainage channels. Both Tillman and Vernon clay loams are best suited for pasture (Goke and Holopeter 1931), as shown by the severely eroded areas over the county where these soils have not been cultivated carefully. In many places, erosion is quite severe although the land has been cultivated little more than 30 years. Foard silt loam belongs to the better productive group of soils and is more suited to cultivation.

\section{CLIMATE}

The average yearly rainfall for Hobart from 1903 to 1930 was 28.13 inches [71 $\mathrm{cm}]$. The months April, May, and June received the most rainfall while December, January, and February proved to be the driest months for those years. The lowest average rainfall came in the year 1910, which was 12.72 inches [32 cm]. The other extreme was 43.33 inches [110 cm] for 1908 (Wahlgren); however, the year 1938 proved a record one for moisture. From January to May, the average precipitation was from an inch to an inch and one-half [2.5-3.75 cm] above the average for each month.

Prevailing winds are from the south in all months except December when they are from the north. The lowest temperature recorded over a period of 28 years is $-11^{\circ} \mathrm{F}$ $\left[-23.9^{\circ} \mathrm{C}\right]$; the highest is $114^{\circ} \mathrm{F}\left[45.6^{\circ} \mathrm{C}\right]$, with an average minimum temperature of $48^{\circ} \mathrm{F}$ 
$\left[8.9^{\circ} \mathrm{C}\right]$ and an average maximum of $74.6^{\circ} \mathrm{F}$ $\left[23.7^{\circ} \mathrm{C}\right]$ for the period. The average date of the last killing frost comes on November 2. There is an average growing season of 213 days.

\section{PREVIOUS COLLECTORS}

Dr. G. G. Shumard (Bull 1932; Eskew 1937) was perhaps the first person to make a collection of plants in this vicinity. He was with Captain R. B. Marcy on his expedition of 1852 to the source of the North Fork of the Red River. The expedition entered the state near the center of the southern border and passed through the Wichita Mountains and into the panhandle of Texas. About 100 plants were collected within the present boundaries of Oklahoma.

Probably the largest single collection was made by the late Dr. G. W. Stevens in 1913 while he was preparing his Flora of Oklahoma. This complete collection is now in the Gray Herbarium at Harvard. Oklahoma Agricultural and Mechanical College has more specimens from the Stevens collection than any other herbarium within the state.

Professor Robert Stratton of Oklahoma Agricultural and Mechanical College has collected in the vicinity to add to his personal herbarium of Leguminosae and for the college herbarium. In 1932, Miss Rotha Bull made a collection of the plants of Greer County which is separated from Kiowa County on the west by North Fork Red River. Mr. C. T. Eskew made a collection of plants in 1937 of the Wichita National Forest within the boundaries of Comanche County which adjoins Kiowa County on the east.

\section{ECOLOGY}

The most common pre-vernal plants are Claytonia virginica L., Glandularia canadensis (L.) Nutt. [=Verbena canadensis], Viola bicolor Pursh [=Viola rafinesquii], V. sororia Willd.
[=V. papilionacea $]$, Erysimum asperum (Nutt.) DC., Anemone caroliniana Walter, A. berlandieri Pritz. $[=A$. decapetala], Lithospermum incisum Lehm. [=Lithospermum angustifolium], Cercis canadensis L., Glandularia bipinnatifida (Nutt.) Nutt. [=Verbena bipinnatifida], Allium canadense L. var. mobilense (Regel) Ownbey [=Allium mutabile], $A$. drummondii Regel $[=A$. nuttallii], Nothascordum bivalve (L.) Britton, and Quincula lobata (Torr.) Raf. [=Physalis lobata]. All of these were to be found on the streams and ravines. The prairie communities have fewer flowering plants; the outstanding ones are Glandularia bipinnatifida, Allium drummondii, Northascordum bivalve, Quincula lobata, Anemone, and Lepidium [=Lepidium apetalum]. Liliaceae and Violaceae have more representatives at this time than other families.

Among the spring plants are Tradescantia obiensis $\mathrm{Raf}$. [=Tradescantia reflexa], T. occidentalis (Britton) Smyth, Baptisia bracteata Muhl. ex Elliott, B. australis (L.) R. Br., Corydalis, Oxalis, and a great percent of Cruciferae, all of which appear on mountains and streams with the budding trees and other woody plants. Cruciferae are in more abundance on the prairies along with Sphaeralcea coccinea (Nutt.) Rydb. [=Malvastrum coccineum], Opuntia bumifusa, Oenothera laciniata Hill, Hordeum pusillium Nutt., Bromus catharticus Vahl. [=Bromus unioloides ], Vulpia octoflora (Walter) Rydb. [=Festuca octoflora], Aristida purpurea Nutt., Yucca glauca Nutt., and Oxalis. Grasses begin flowering in late spring and early summer when they become predominant.

Leguminosae is another family which flowers mainly in the summer. Other plants which become predominant at this time are Argemone albiflora Hornem. [= Argemone alba], A. polyanthemos (Fedde) G.B. Ownbey $[=A$. intermedia], Cirsium [=Cirsium discolor], Centaurea americana Nutt., Gaillardia, Tribulus terrestris L., Plantago patagonica Jacq. [=Plantago purshii], Krameria lanceolata Torr. [=Krameria secundiflora], Erigeron strigosus Muhl. ex Willd. 
[=Erigeron ramosus], Chloris verticillata Nutt., Solanum elaeagnifolium Cav., S. rostratum Dunal, Polygonum, Salsola tragus L. [=Salsola kali], and the greatest percent of Euphorbiaceae.

During the latter part of the summer the composites begin to gain predominance as for number of species in flower, but the grass family is still the most important as to the amount of space it covers. During the autumn the outstanding plants are Helianthus, Rhus, Vernonia, Euphorbia marginata Pursh, Liatris punctata Hook., Solidago, Aster, Ambrosia, Xanthium, Sorghastrum nutans (L.) Nash, and others of the tall grass group.

Annual and biennial plants on the mountains and streams are not so different from those of the prairies in the summer and fall as they are in the spring. The fall grasses are more adapted to the former habitat. Many woody plants are seeding in the autumn, and in the latter part of the year the mountainsides are colorful with the brilliant foliage of the trees and shrubs.

Along the streams, trees and woody plants are dominant. Carya illinoinensis, Juglans nigra L., Rhus glabra L., Ulmus americana, Vitis, Fraxinus, Toxicodendron, Sapindus saponaria L. var drummondii (Hook. \& Arn.) L.D. Benson [=Sapindus drummondii], and Salix nigra Marshall are the outstanding plants in this type of vegetation. These same genera are found on the mountains, but species of Quercus become dominant in the eastern section of the county. Other mountain plants are Rhus aromatica Aiton $[=R h u s$ trilobata], Ptelea trifoliata L., Ribes aureum Pursh, Baptisia, Sedum, Ceanothus americanus L., Rubus, Galium aparine L., and Poa arachnifera Torr.

Plants in dry sand and along the rivers form another distinctly different type. Artemisia, Mentrelia, Sporobolus, and species of Prunus form the dominant covering on the sand hills, and Tamarix [=Tamarix gallica] is found in abundance in damp sandy soil. Other outstanding plants here are
Glandularia canadensis, Comandra umbellata (L.) Nutt. ssp. pallida (A. DC.) Piehl [=Comandra pallida], Lithaspermum incisum, Cenchrus, and a species of Gaura.

The types of vegetation mentioned above are all connected by the prairie type which covers the greatest percent of the area of the county. Here is found one main association in the undisturbed pastures. Prosopis glandulosa var. glandulosa forms an orchard type of growth, and under the trees the dominant vegetation is Bouteloua dactyloides (Nutt.) J.T. Columbus [=Buchloe dactyloides] interspersed with Opuntia bumifusa.

\section{SUMMARY}

The Flora of Kiowa County, Oklahoma includes six species of pteridophytes, one species of gymnosperms, and 489 species of angiosperms. More than one third of these are in Compositae, Gramineae, and Leguminosae. There are 81 families represented. The 11 largest families, with the number of species, are Compositae, 86; Gramineae, 58; Leguminosae, 41; Onagraceae, 17; Euphorbiaceae, 16; Cruciferae, 16; Polygonaceae and Solanaceae, 12 each; and Asclepiadaceae, Cyperaceae, and Labiatae, 11 each. The three largest families comprise 37.4 per cent of the total number of species.

The county lies in the plains region. The prairies are broken by the Wichita Mountains and a few streams, of these the North Fork Red River is the largest. The vegetation is mainly that adapted to the prairies. Tall grass is found near mountains or streams; it is predominately a short-grass area. Trees are to be seen along streams or on mountainsides. The only native trees on the prairies are mesquite (Prosopis glandulosa var. glandulosa) which grow in association with cactus (Opuntia humifusa) and buffalo grass (Bouteloua dactyloides). 


\section{ACKNOWLEDGEMENTS}

The writer wishes to express her appreciation to the following people for their services in preparing this paper: Dr. H. I. Featherly of the Oklahoma Agricultural and Mechnical College, under whose direction this study was made, for constant advice and criticism; Dr. K. Starr Chester, head of the Department of Botany, and Professor R. H. Stratton, of the same department, for their aid in securing materials; and Dr. Elbert L. Little, Jr., in the United States Forest Service at Flagstaff, Arizona, for suggestions. The writer also wishes to express her appreciation to her family for assistance in collecting and preparing specimens.

\section{BIBLIOGRAPHY}

Britton, N.L. and A. Brown. 1913. An Illustrated Flora of the Northern United States, Canada, and the British Possessions. $2^{\text {nd }}$ ed. 3 volumes. New York: [Charles Scribner and Sons].

Bull, R.Z. 1932. V ascular Plants of Greer County, Oklahoma [Master's thesis]. Norman (OK): University of Oklahoma.

Eskew, C.T. 1937. Flowering Plants of the Wichita National Forest [Master's thesis]. Norman (OK): University of Oklahoma.

Featherly, H.I. 1938. Grasses of Oklahoma. Oklahoma Agricultural Experiment Station Technical Bulletin No. 3. Stillwater (OK): Oklahoma Agricultural and Mechanical College.

Featherly, H.I. and E.E. Still. 1934. The Ferns of Oklahoma. Botanical Studies No. 1. Experiment Station Circular No.
80. Stillwater (OK): Oklahoma Agricultural and Mechanical College.

Goke, A.W. and C.A. Holopeter. 1931. Soil Survey of Kiowa County, Oklahoma. United States Department of Agriculture Bulletin No. 14.

Hitchock, A.S. 1935. Manual of the Grasses of the United States. Misc. Pub. No. 200. Washington (DC): Government Printing Office.

Jeffs, R.E. and E.L. Little, Jr. A Preliminary List of the Ferns and Seed Plants of Oklahoma. University of Oklahoma Biological Survey, Vol. 11, No. 2.

Little, E.L., Jr. Flora of Muskogee County, Oklahoma. 1938. The American Midland Naturalist 19:369-389.

Mattoon, W.R. and G.G. Phillips. 1936. Forest Trees of Oklahoma. Oklahoma Forest Commission Publication No. 1. Reprint No. 4. United States Department of Agriculture.

Robinson, B.L. and M.L. Fernald. 1908. Gray's New Manual of Botany. $7^{\text {th }}$ ed. New York: American Book Company.

Rydberg, P.A. 1932. Flora of the Prairies and Plains of Central North America. New York: [New York Botanical Garden].

Sawyer, R.W. 1929. Kiowa and Washita counties, Oklahoma. Geological Survey Bulletin $40 \mathrm{HH}$.

Small, J.K. 1913. Flora of the Southeastern United States. $2^{\text {nd }}$ ed. New York: [published by author].

Stemen, T.R. and W.S. Meyers. 1937. Oklahoma Flora. Oklahoma City: Harlow.

Wahlgren, H.F. [date unknown]. Climatological Data. United States Department of Agriculture. Weather Bureau. Oklahoma City. 


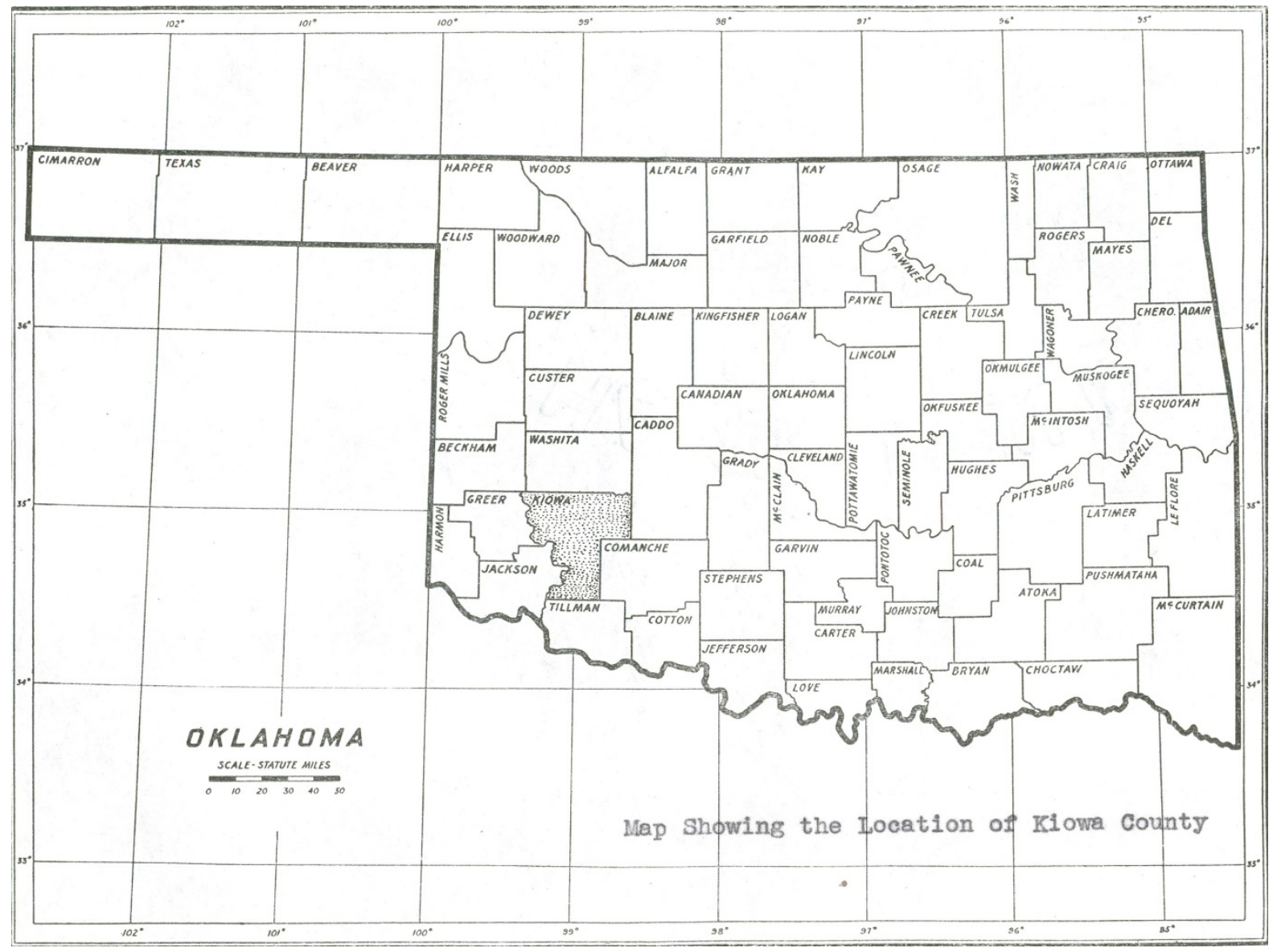

Figure Map of Kiowa County, Oklahoma 


\section{APPENDIX A}

\section{List of Species, Kiowa County, OK}

[Nomenclature has been updated using the PLANTS Database (http://plants.usda.gov/plants).]

\section{PTERIDOPHYTA}

Dryopteridaceae [Polypodiaceae]

Woodsia obtusa (Spreng.) Torr.

Marsileaceae

Marsilea vestita Hook. \& Grev.

Pteridaceae [Polypodiaceae]

Cheilanthes eatonii Baker

Cheilanthes lanosa (Michx.) D.C. Eaton

[=Cheilanthes lanulosa (Michx.) Watt]

Notholaena standleyi Maxon

Pellaea atropurpurea (L.) Link blunt-lobed woodsia

water fern, hairy pepperwort low places, pastures

Eaton's lip fern

hairy lip fern

Standley's notholaena

purple cliff brake mountainsides

mountainsides

mountainsides

mountainsides

mountainsides

\section{SPERMATOPHYTA}

\section{Gymnosperms}

\section{Cupressaceae [Pinaceae]}

Juniperus virginiana $\mathrm{L}$.

red cedar

hillsides

\section{Angiosperms}

\section{Acanthaceae}

Ruellia pedunculata Torr. ex A. Gray

Ruellia sp.

[=Ruellia ciliosa Pursh, misapplied]

stalked ruellia

mountainsides; summer, fall

hairy ruelllia

rivers; summer, fall

yucca, bear-grass, soap weed

pastures, roadsides;

spring

Aizoaceae

Mollugo verticillata $\mathrm{L}$.

carpet-weed

pastures, common;

summer

prostrate amaranth

fields, pastures, common; summer 
Amaranthus hybridus L.

Amaranthus retroflexus $\mathrm{L}$.

Amaranthus tuberculatus (Moq.) Sauer

\section{Anacardiaceae}

Rhus aromatica Aiton

[=Rhus canadense Mill., Rhus trilobata Nutt.]

Rhus glabra L.

Toxicodendron radicans (L.) Kuntze

\section{Apocynaceae \\ Amsonia tabernaemontana Walter \\ Apocynum cannabinum L.}

\section{Asclepiadaceae}

Asclepias amplexicaulis Sm.

Asclepias asperula (Decne.) Woodson ssp. capricornu (Woodson) Woodson [=Asclepiodora decumbens (Nutt.) A. Gray] Asclepias engelmanniana Woodson [=Acerates auriculata Engelm. ex Torr.] Asclepias latifolia (Torr.) Raf.

Asclepias stenophylla A. Gray [incl. Acerates angustifolia (Nutt.) Decne.] Asclepias tuberosa L.

Asclepias verticillata $\mathrm{L}$.

Asclepias viridiflora Raf. [=Acerates viridiflora (Raf.) Pursh ex Eaton] Asclepias viridis Walter

[=Asclepiodora viridis (Walter) A. Gray] Gonolobus suberosus (L.) R. Br.

[=Vincetoxicum gonocarpos Walter]

\section{Boraginaceae}

Heliotropium convolvulaceum (Nutt.) A. Gray Heliotropium indicum L. dark green pig-weed

red root

western water-hemp

fragrant sumac, sumac

smooth upland sumac

poison ivy

broad-leaved amsonia

dogbane, Indian hemp

milkweed, silkweed

milkweed

green milkweed

broad-leaved milkweed

narrow-leaved milkweed

butterfly weed

whorled milkweed

green milkweed

oblong-leaved milkweed

large-leaved angle-pod

sand heliotrope

Indian heliotrope pastures; spring to

fall

roadsides; spring, summer

pastures; summer

mountains, streams; spring, summer creeks, hillsides; common; summer mountainsides, streams; summer

mountain ravines; spring roadsides; common; summer

near rivers; spring, summer mountainsides; spring

roadsides; summer

dry sandy soils; summer prairies; summer

sand, near rivers; spring, summer mountainsides; spring, summer prairies; summer

prairies; summer rivers; early summer

rivers; late summer pastures, creeks; spring to fall 
Lappula occidentalis (S. Watson)

Greene [=Lappula redowskii (Hornem.)

Greene var. occidentalis (S. Watson) Rydb.]

Lithospermum incisum Lehm.

[=Lithospermum angustifolium Michx.]

Myosotis verna Nutt.

[=Myosotis virginica (L.) Britton, Sterns \&

Poggenb., misapplied]

Onosmodium bejariense DC. ex A. DC.

[=Onosmodium occidentale Mack.]

\section{Cactaceae}

Echinocereus reichenbachii (Terscheck ex

Walp.) J.N. Haage

[=Echinocereus caespitosus (Englem.) Engelm.

Opuntia humifusa (Raf.) Raf.

\section{Campanulaceae [incl. Lobeliaceae]}

Lobelia cardinalis L.

Lobelia spicata Lam. var. leptostachys

(A. DC.) Mack. \& Bush

[=Lobelia leptostachys A. DC.]

Triodanis leptocarpa (Nutt.) Nieuwl.

[=Specularia leptocarpa (Nutt.) A. Gray]

Triodanis perfoliata (L.) Nieuwl.

[=Specularia perfoliata (L.) A. DC.]

\section{Capparaceae}

Cleome serrulata Pursh

Cleomella angustifolia Torr.

\section{Caprifoliaceae}

Symphoricarpos orbiculatus Moench

Viburnum rufidulum Raf.

\section{Caryophyllaceae [incl. Illecebraceae]}

Cerastium brachypodum (Engelm. ex A. Gray)

B.L. Rob.

Cerastium nutans Raf.

Paronychia jamesii Torr. \& A. Gray

Silene antirrhina $\mathrm{L}$.

Stellaria media (L.) Vill. western stick-weed

mountainsides;

spring

puccoon

spring or early scorpion-grass

western false gromwell

dry sandy soils;

spring

sand near rivers;

spring

prairies; summer

lace cactus

mountainsides

western prickly-pear

pastures; common; spring

cardinal flower, red lobelia

spiked lobelia

mountain ravines;

summer

mountainsides;

summer

western Venus's looking-glass

Venus's looking-glass

summer

prairies, streams;

spring, summer

pink cleome, stinking clover

prairies; summer

coral-berry, Indian currant

southern black-haw

streams; summer mountainsides;

spring

prairies; spring

mountainsides;

spring

James's whitlow-wort

sleepy catchfly

common chickweed, starwort prairies; summer roadsides; spring low damp places; early spring 


\author{
Chenopodiaceae \\ Chenopodium album L. \\ Cycloloma atriplicifolium (Spreng.) J.M. Coult. \\ Monolepis nuttalliana (Schult.) Greene \\ Salsola tragus $\mathrm{L}$. \\ [=Salsola kali L., misapplied] \\ Commelinaceae \\ Commelina erecta $\mathrm{L}$. \\ [incl. Commelina crispa Woot.] \\ Commelina virginica $\mathrm{L}$. \\ [incl. Commelina hirtella Vahl] \\ Tradescantia occidentalis (Britton) Smyth
}

lamb's quarters

sand tumbleweed

monolepis

Russian thistle

slender day-flower, crinkle-leaved day-flower

Virginia day-flower, bearded day-flower western spiderwort, trinity

common yarrow, wooly common yarrow

ragweed

western ragweed

great ragsweed

August flower kindling-weed sand-daisy

white-flowered sand-daisy dark-leaved mugwort

willow baccharis

Texas berlandiera

false boneset

centaurea

chaetopappa

aster

Nuttall's golden aster

hispid golden aster roadsides, common; summer rivers; summer common near dwellings; spring roadsides, cultivated soil; summer

mountainsides, creeks; spring, summer, fall mountain ravines, streams; summer mountainsides; spring

roadsides, prairies, creeks; summer ravines; summer, fall roadsides, pastures; fall creeks; summer, fall roadsides; fall hillsides; summer

rivers; summer dry hillsides near rivers; late summer rivers; early summer streams; summer

prairies; summer, fall roadsides; summer rivers; spring hillsides; summer

mountainsides; summer, fall rivers; summer

Chrysopsis sp. [=Chrysopsis villosa (Pursh) Nutt. ex DC. var. hispida (Hook.) A. Gray, misapplied] 


\section{Cirsium ochrocentrum A. Gray}

Cirsium sp.

[=Cirsium discolor (Muhl. ex Willd.) Spreng., misapplied]

Conyza canadensis (L.) Cronquist

[=Erigeron canadensis L.]

Coreopsis grandiflora Hogg ex Sweet

Coreopsis tinctoria Nutt.

Coreopsis sp.

[=Coreopsis verticillata L., misapplied]

Dracopis amplexicaulis (Vahl) Cass.

[=Rudbeckia amplexicaulis Vahl]

Echinacea angustifolia DC.

[=Brauneria angustifolia (DC.) A. Heller]

Engelmannia peristenia (Raf.) Goodman \&

C.A. Lawson

[=Engelmannia pinnatifida A. Gray ex. Nutt.]

Erigeron strigosus Muhl. ex Willd.

[=Erigeron ramosus (Walter) Britton, Sterns

\& Poggenb.]

Euthamia gymnospermoides Greene

Evax prolifera Nutt. ex DC.

Evax verna Raf.

[=Evax multicaulis DC.]

Flaveria campestris J.R. Johnst.

Gaillardia pulchella Foug.

Gaillardia suavis (A. Gray \& Engelm.) Britton

\& Rusby

Gaillardia sp.

[=Gaillardia aristata Pursh, misapplied]

Grindelia papposa G.L. Nesom \& Suh

[=Haplopappus ciliatus (Nutt.) DC.]

Grindelia squarrosa (Pursh) Dunal

Helenium amarum (Raf.) H. Rock var. amarum [=Helenium tenuifolium Nutt.]

Helenium amarum (Raf.) H. Rock var. badium

(A. Gray ex S. Watson) Waterf.

[=Helenium tenuifolium Nutt. var. badium

A. Gray ex S. Watson] yellow-spined thistle

field thistle

horsetail, horse-weed

large-flowered coreopsis

golden coreopsis,

garden tickseed

whorled tickseed

cone flower

narrow-leaved purple

cone-flower

engelmannia

daisy fleabane

viscid bushy goldenrod

rabbit tobacco

rabbit tobacco

plains flaveria

showy gaillardia

cut-leaved rayless

three-nerved gaillardia

gaillardia

rosin-weed

broad-leaved gum plant, rosin-weed

fine-leaved sneezeweed

fine-leaved sneezeweed prairie pastures;

summer

roadsides; common;

summer

pastures; summer, fall

rivers; summer

streams; spring,

summer

rivers; late summer

streams; late spring

hillsides; spring to

fall

hillsides; summer

pastures; spring

prairies; late

summer

roadsides, pastures;

spring, summer

roadsides, pastures;

common; spring,

early summer

dry plains; late

summer

hillsides; summer,

fall

prairie roadsides;

spring

roadsides; common;

summer

prairie roadsides;

spring to fall

rivers; summer, fall

creeks; summer

streams; summer 


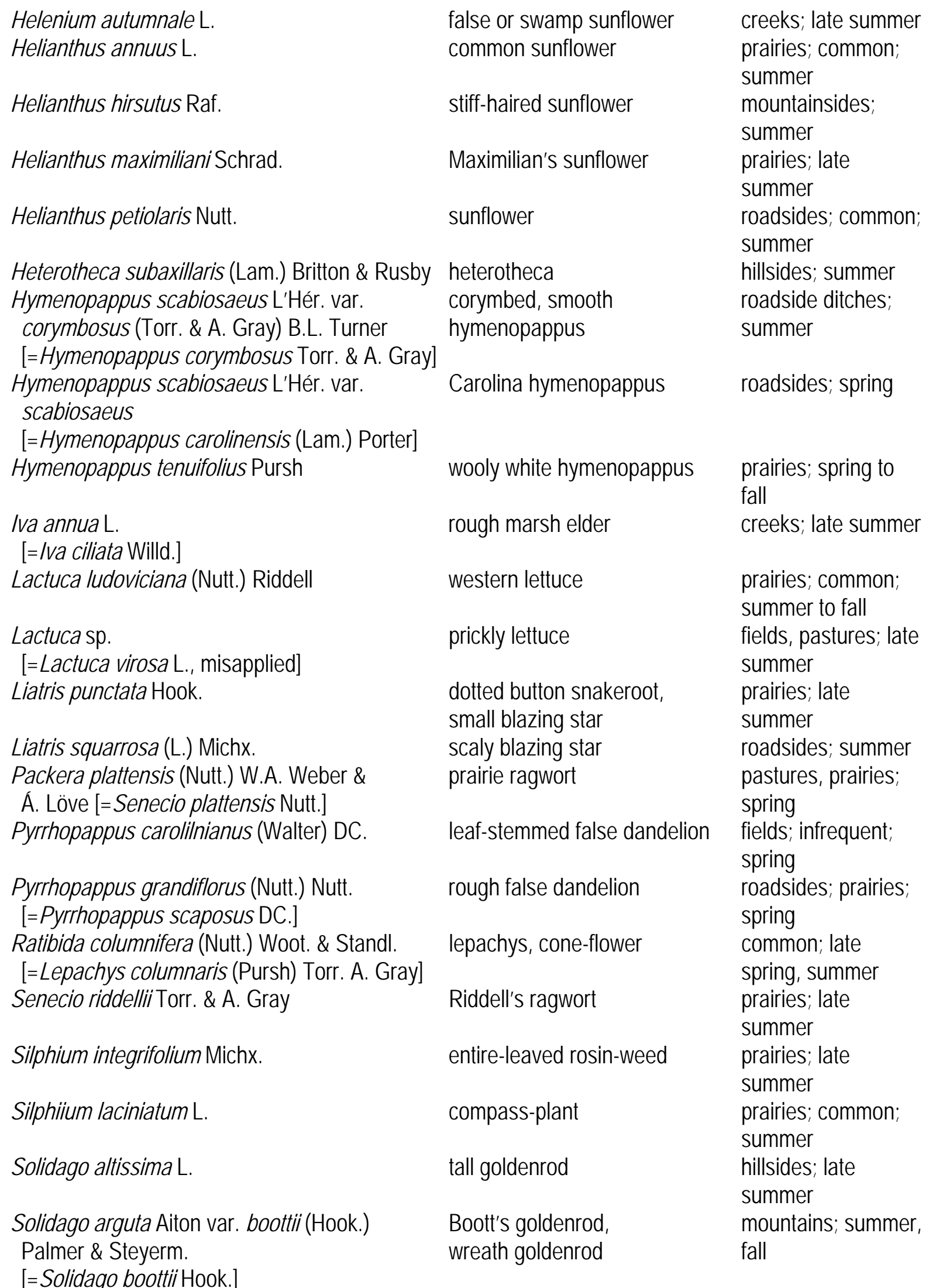


Solidago gigantea Aiton [=Solidago serotina Aiton]

Solidago missouriensis Nutt.

Solidago petiolaris Aiton

Solidago radula Nutt.

Sonchus asper (L.) Hill

Symphyotrichum divaricatum (Nutt.)

G.L. Nesom [Aster exilis Elliott]

Symphyotrichum ericoides (L.) G.L. Nesom [=Aster multiflorus Aiton]

Symphyotrichum falcatum (Lindl.) G.L.

Nesom var. commutatum (Torr. \& A. Gray)

G.L. Nesom [=Aster commutatus

(Torr. \& A. Gray) A. Gray]

Symphyotrichum fendleri (A. Gray) G.L.

Nesom [=Aster fendleri A. Gray]

Taraxacum officinale F.H. Wigg.

[=Taraxacum vulgare Lam.]

Tetraneuris linearifolia (Hook.) Greene

[=Actinea linearifolia (Hook.) Kuntze]

Tetraneuris scaposa (DC.) Greene

[=Actinea scaposa (DC.) Kuntze var. linearis

(Nutt.) B.L. Rob.]

Thelesperma filifolium (Hook.) A. Gray

[=Thelesperma trifidum (Poir.) Britton]

Thelesperma megapotamicum (Spreng.)

Kuntze [=Thelesperma gracile (Torr.) A. Gray]

Vernonia baldwinii Torr.

Vernonia gigantea (Walter) Trel.

[=Vernonia altissima Nutt.]

Vernonia missurica Raf.

Xanthisma texanum DC.

Xanthium strumarium L.

[=Xanthium pensylvanicum Wallr.,

Xanthium speciosum Kearney]

\section{Convolvulaceae}

Cuscuta cephalanthi Engelm.

Cuscuta cuspidata Engelm.

Cuscuta indecora Choisy

Evolvulus nuttallianus Schult.

[Evolvulus argenteus Pursh] late goldenrod

creeks; late summer

Missouri goldenrod

downy ragged goldenrod

western rough goldenrod

spiny sow-thistle

slim aster

many-flowered aster

aster

mountainsides;

summer

fields; fall

prairies; summer

roadsides; spring to

fall

creeks; summer

prairies; summer,

fall

prairies; spring

Fendler's aster

pastures; summer

common dandelion

fine-leaved actinea

narrow-leaved actinea

rivers; summer

thelesperma, tickseed

rayless thelesperma

fields, pastures; spring to fall

hillsides; summer

Baldwin's ironweed

tall ironweed

Missouri ironweed

Texas xanthisma, sleepy daisy

cocklebur, great cocklebur

button-bush dodder

cuspidate dodder

pretty dodder

pastures; ragweeds;

dwarf morning-glory parasite; summer

summer

on composites;

summer

prairies, mountains; common; summer

to fall

prairies; summer

rivers; summer

streams; summer

prairies; summer

roadsides, ravines; common; summer, fall

prairies; summer prairies; fall 
Ipomoea leptophylla Torr.

Ipomoea pandurata (L.) G. Mey.

\section{Cornaceae}

Cornus drummondii C.A. Mey.

[=Cornus asperifolia Michx., misapplied]

Cornus florida $\mathrm{L}$.

\section{Crassulaceae}

Sedum nuttallianum Raf.

\section{Cruciferae}

Capsella bursa-pastoris (L.) Medik.

Descurainia pinnata (Walter) Britton

[=Sisymbrium canescens Nutt.]

Descurainia sp.

[=Sisymbrium incisum Englem. ex A. Gray, misapplied]

Dimorphocarpa candicans (Raf.) Rollins [=Dithyrea wislizeni Engelm.]

Draba brachycarpa Nutt. ex Torr. \& A. Gray

Draba cuneifolia Nutt. ex Torr. \& A. Gray

Erysimum asperum (Nutt.) DC.

Lepidium virginicum $\mathrm{L}$.

Lepidium sp.

[=Lepidium apetalum Willd., misapplied]

Lesquerella auriculata (Engelm. \& A. Gray)

S. Watson

Lesquerella densiflora (A. Gray) S. Watson

Lesquerella ovalifolia Rydb. ex Britton

Nasturtium officinale W.T. Aiton

[=Radicula nasturtium-aquaticum (L.)

Britten \& Rendle]

Rorippa sessiliflora (Nutt.) Hitchc. [=Radicula sessiflora (Nutt.) Greene]

Sibara virginica (L.) Rollins

[=Arabis virginica (L.) Poir.]
Nuttall's stonecrop

rough-leaved dogwood

flowering dogwood

streams; spring

mountainsides;

spring

rocks on

mountainsides;

spring

roadsides; early

summer

roadside ditches;

summer

fields, meadows;

spring

hillsides; spring

western tansy-mustard

prairie roadsides; spring, summer

spectacle pod

dry, sandy soils, near rivers; summer

short-fruited whitlow-grass

wedge-leaved whitlow-grass

yellow phlox

wild pepper grass

wild pepper grass

hairy bladder-pod

bladder-pod

slender bladder-pod

water cress

sessile-flowered cress

cut-leaved rock-cress fields, pastures;

early spring

fields; common;

early spring mountainsides;

spring

abundant; spring roadsides; common; spring prairies; early spring

near rivers; spring rocky hillsides; spring streams; spring

mountain ravines; spring mountainsides; early spring 


\section{Cucurbitaceae}

Cucurbita foetidissima Kunth

[=Pepo foetidissima (Kunth) Britton]

\section{Cyperaceae}

Carex vulpinoidea Michx.

Cyperus echinatus (L.) Alph. Wood

[=Cyperus ovularis (Michx.) Torr.]

Cyperus esculentus L.

Cyperus odoratus L.

[=Cyperus ferax Rich.]

Cyperus strigosus L.

Eleocharis compressa Sull.

Fuirena simplex Vahl

Lipocarpha micrantha (Vahl) G. Tucker

[=Hemicarpha micrantha (Vahl) Pax]

Schoenoplectus americanus (Pers.) Volkart

ex Schinz \& R. Keller

[=Scirpus americanus Pers.]

Scirpus pendulus Muhl.

[=Scirpus lineatus, misapplied]

\section{Ebenaceae}

Diospyros virginiana L.

\section{Euphorbiaceae \\ Cnidoscolus texanus (Müll. Arg.) Small \\ [=Jatropha stimulosa Michx.] \\ Croton capitatus Michx. \\ Croton glandulosus L. var. septentrionalis \\ Müll. Arg. \\ Croton lindheimerianus Scheele \\ Croton texensis (Klotzsch) Müll. Arg. \\ Euphorbia dentata Michx. \\ Euphorbia spathulata Lam. \\ [=Euphorbia dictyosperma Fisch. \& C.A. Mey.] \\ Euphorbia geyeri Engelm. \& A. Gray \\ Euphorbia maculata L. \\ Euphorbia marginata Pursh \\ Euphorbia missurica Raf. \\ [=Euphorbia petaloidea Engelm.]}

Missouri gourd

fields, streams;

summer

fox sedge

globose cyperus

yellow nut-grass

coarse cyperus

straw colored cyperus

flat-stemmed spike-rush

western-umbrella-grass

dwarf sedge

three-cornered bulrush,

sand-bar bulrush

reddish bulrush

persimmon

spurge nettle

goat-weed, hogwort

Lindheimer's croton-weed

Texas croton

toothed spurge

reticulate-seeded spurge

Geyer's spurge

spurge

snow-on-the-mountain

white-flowered spurge mountain ravines;

spring

mountain ravines;

summer

rivers

rivers

damp soils, pastures

mountain ravines

ponds; summer

rivers; late summer

riversides; summer

damp ravines;

summer

damp ravines;

summer

rivers; spring, summer roadsides; spring to fall

creeks; summer

pastures; summer roadsides; midsummer streams; summer plains; summer

rivers; summer prairies; spring to fall hillsides, rivers; summer, fall prairies; summer 
Euphorbia nutans Lag.

[=Euphoribia preslii Guss.]

Euphorbia serpens Kunth

Stillingia sylvatica L.

Tragia ramosa Torr.

Tragia sp.

[=Tragia nepetifolia Cav., misapplied]

\section{Fagaceae}

Quercus fusiformis Small

[=Quercus virginiana Mill., misapplied]

Quercus macrocarpa Michx.

Quercus marilandica Münchh.

Quercus muehlenbergii Engelm.?

[=Quercus prinus L.]

Quercus shumardii Buckley var. schneckii (Britton) Sarg.

Quercus stellata Wangenh.

\section{Fumariaceae}

Corydalis aurea Willd.

Corydalis micrantha (Engelm. ex A. Gray)

A. Gray [=Corydalis campestris (Britton)

J. Bucholz \& Palmer]

\section{Gentianaceae}

Eustoma exaltatum (L.) Salisb. ex G. Don

[=Eustoma russellianum (Hook.) G. Don]

Sabatia angularis (L.) Pursh

Sabatia campestris Nutt.

Geraniaceae

Geranium carolinianum L.

\section{Gramineae}

Agrostis hyemalis (Watt) Britton, Sterns \&

Poggenb.

Alopecurus geniculatus L.

Andropogon gerardii Vitman

[=Andropogon furcatus Muhl. ex Willd.] large spotted spurge,

upright spotted spurge

mountains, spring to

round-leaved spreading spurge

queen's delight

branching tragia

catnip-leaved tragia

fall

prairies; spring to

fall

prairies; spring to

fall

mountainsides;

summer

rivers; summer

live oak

mountains;

pre-vernal

bur oak, mossy-cup oak

black jack oak

mountainsides;

pre-vernal

mountains;

pre-vernal

cow oak, swamp oak

mountains; spring

Schneck's red oak,

mountains; spring

spotted oak

post oak

mountains;

pre-vernal

golden corydalis

plains corydalis

prairies; spring

creeks, pastures, near moisture;

spring

Russell's eustoma

creeks; summer

rose pink, bitter bloom

prairie sabatia

creeks; summer

prairies; summer

wild geranium

mountains, streams; spring

mountainsides

foxtail

streams

forked beard-grass,

mountainsides 
Aristida dichotoma Michx.

Aristida oligantha Michx.

Aristida purpurascens Poir.

Aristida purpurea Nutt.

Bothriochloa laguroides (DC.) Herter ssp.

torreyana (Steud.) Allred \& Gould

Bouteloua curtipendula (Michx.) Torr.

Bouteloua dactyloides (Nutt.) J.T. Columbus

[=Buchlöe dactyloides (Nutt.) Engelm.]

Bouteloua gracilis (Willd. ex Kunth) Lag.

ex. Griffiths

Bouteloua hirsuta Lag.

Bouteloua sp.

[=Bouteloua breviseta Vasey, not in OK]

Bromus arvensis $\mathrm{L}$.

Bromus catharticus Vahl

[=Bromus unioloides Kunth]

Bromus racemosus L.

[=Bromus commutatus Schrad.]

Cenchrus spinifex Cav.

[=Cenchrus pauciflorus Benth.]

Chasmanthium latifolium (Michx.) Yates

[=Uniola latifolia Michx.]

Chloris verticillata Nutt.

Cynodon dactylon (L.) Pers.

Dichanthelium acuminatum (Sw.) Gould \&

C.A. Clark [=Panicum tennesseense Ashe]

Digitaria cognata (Schult.) Pilg.

[=Leptoloma cognata (Schult.) Chase]

Digitaria sanguinalis (L.) Scop.

Echinochloa crus-galli (L.) P. Beauv.

Eleusine indica (L.) Gaertn.

Elymus canadensis L.

Elymus glabriflorus (Vasey ex L.H. Dewey)

Scribn. \& C.R. Ball [=E. virginicus L. var.

glabriflorus (Vasey) Bush]

Elymus repens (L.) Gould

[=Agropyron repens (L.) P. Beauv.]

Eragrostis capillaris (L.) Nees

Eragrostis cilianensis (All.) Vign. ex Janchen

Eragrostis curtipedicellata Buckley

Eragrostis secundiflora J. Presl

Eragrostis trichodes (Nutt.) Alph. Wood aristida

few-flowered aristida

purplish aristida

purple three-awn

andropogon

fall grama-grass

buffalo grass

blue grama-grass

hairy mesquite-grass

bouteloua

field chess

brome grass

brome grass

field sandbur

broadleaf uniola

windmill grass

Bermuda grass

panicum

diffuse crag-grass

large crab-grass

barnyard grass

goosegrass

Canada wild-rye

Virginia wild-rye

couch grass

lace-grass

stinkgrass

short-stalked love-grass

love-grass

eragrostis prairies; summer

pastures; summer

pastures, roadsides

prairies; spring

creeks; summer

prairies; summer

pastures; summer

pastures; summer, fall

pastures; summer

creeks; spring,

summer

roadsides; summer

pastures, roadsides;

spring

roadsides; common;

summer

rivers; summer

mountains; autumn

prairies; common;

spring

roadsides; common

mountain ravines

fields; fall

fields

streams

pastures

streams, ravines

streams

fields; summer

prairies; summer

fields, roadsides;

summer

roadsides, pastures;

summer

near river; summer

near river; summer 
Erioneuron pilosum (Buckley) Nash

[=Triodia pilosa (Buckley) Merr.]

Hordeum pusillum Nutt.

Melica nitens (Scribn.) Nutt. ex Piper

Panicum anceps Michx.

Panicum capillare L.

Panicum dichotomiflorum Michx.

Panicum obtusum Kunth

Panicum rigidulum Bosc ex Nees

[=Panicum agrostoides Spreng.]

Panicum virgatum $\mathrm{L}$.

Pascopyrum smithii (Rydb.) Á. Löve

[=Agropyron smithii Rydb.]

Paspalum setaceum Michx.

[=Paspalum pubescens Muhl. ex Willd.]

Phalaris caroliniana Walter

Poa arachnifera Torr.

Schedonnardus paniculatus (Nutt.) Trel.

Schizachyrium scoparium (Michx.) Nash

Setaria parviflora (Poir.) Kerguélen

[=Setaria geniculata (Willd.) P. Beauv.]

Setaria pumila (Poir.) Roem. \& Schult. [=Setaria lutescens (Weigel) F.T. Hubbard]

Setaria viridis (L.) P. Beauv.

Sorghastrum nutans (L.) Nash

Sorghum halepense (L.) Pers.

Sphenopholis obtusata (Michx.) Scribn. Sporobolus cryptandrus (Torr.) A. Gray

Tridens flavus (L.) Hitchc.

[=Triodia flava (L.) Smyth]

Vulpia octoflora (Walter) Rydb.

[=Festuca octoflora]

\section{Grossulariaceae [Saxifragaceae]}

Ribes aureum Pursh hairy triodia

little barley

three-flower melic

panicum

witch-grass, tumbleweed

fall panicum

blunt panic-grass, range-grass

panicum

switch-grass, wild red-top

western wheat-grass

paspalum

Carolina canary-grass

Texas blue grass

Texas crab-grass, wire-grass

prairie beard-grass

knot-root bristle-grass

yellow fox-tail

green foxtail-grass

Indian-grass

Johnson grass

prairie wedge grass

sand dropseed

purpletop

six-weeks fescue

Missouri or buffalo currant mountainsides;

spring

prairies; spring

mountainsides;

spring

rivers

fields; summer

streams; fall

rivers

streams

creeks; fall

prairies; spring,

summer

along rivers

moist places,

roadsides; spring

highways, hillsides;

spring

prairies; common;

summer

roadsides

creeks, roadsides;

spring

roadsides; common;

late spring

fields; summer

mountains; fall

roadside ditches;

summer

streams

rivers; summer, fall

mountains, ravines

fields, pastures;

spring

hillsides, streams;

spring

hairy phacelia

small-flowered phacelia prairies; spirng

prairies; spring

Phacelia sp.

[=Phacelia dubia (L.) Trel., misapplied] 


\section{Iridaceae}

Sisyrinchium angustifolium Mill. [incl. Sisyrinchium gramineum Curtis]

Sisyrinchium langloisii Greene

[=Sisyrinchium varians E.P. Bicknell]

\section{Juglandaceae}

Carya illinoinensis (Wagenh.) K. Koch Juglans microcarpa Berl.

[=Juglans rupestris Engelm. ex Torr.]

Juglans nigra L.

\author{
Juncaceae \\ Juncus biflorus Elliott \\ [=Juncus aristulatus Michx.] \\ Juncus brachycarpus Engelm. \\ Juncus interior Wiegand \\ Juncus marginatus Rostk. \\ Juncus torreyi Coville \\ Krameriaceae [Leguminosae] \\ Krameria lanceolata Torr. \\ [=Krameria secundiflora DC., misapplied]

\section{Labiatae} \\ Hedeoma hispida Pursh \\ Lamium amplexicaule $\mathrm{L}$. \\ Monarda citriodora Cerv. ex Lag. \\ [=Monarda dispersa Small] \\ Monarda fistulosa L. \\ Monarda punctata L. \\ Salvia azurea Michx. ex Lam. var. grandiflora \\ Benth. \\ Salvia reflexa Hornem. \\ [=Salvia lancaefolia Poir.] \\ Scutellaria drummondii Benth. \\ Scutellaria wrightii A. Gray \\ Teucrium canadense $\mathrm{L}$.
}

blue-eyed grass, stout blue-eyed grass

variable blue-eyed grass

pecan

little walnut

black walnut

large grass-leaved rush

rush

Indian rush

awn-petaled rush

Torrey's rush

bank-bur rough or little pennyroyal

henbit, dead nettle

purple lemon mint

horse mint, wild bergamot

horse mint

tall blue sage

lance-leaved sage

Drummond's skullcap

Wright's skullcap

germander, wood sage creeks, prairies; spring

prairies; spring

streams; spring

creeks; spring

creeks; spring

riversides

roadside ditches;

summer

roadside ditches;

summer

rivers; summer

rivers; summer

prairie roadsides; common; summer

plains; summer fields, roadsides; early spring praries; summer

ravines; early summer dry sandy soils; summer plains; spring, summer creeks; spring, summer roadside ditches; common; spring hillsides; spring ravines; summer 


\section{Leguminosae}

Acacia angustissima (Mill.) Kuntze acacia

Amopha canescens Pursh

Amorpha fruticosa $\mathrm{L}$.

Astragalus canadensis L.

Astragalus crassicarpus Nutt.

[=Astragalus caryocarpus Ker Gawl.]

Astragalus lotiflorus Hook.

Astragalus nuttallianus DC.

Baptisia australis (L.) R. Br.

Baptisia bracteata Muhl. ex Elliott

Cercis canadensis L.

Chamaecrista fasciculata (Michx.) Greene

[=Cassia chamaecrista L.]

Dalea aurea Nutt. ex Pursh

[=Parosela aurea (Nutt. ex Pursh) Britton]

Dalea candida Michx. ex Willd.

[=Petalostemon candidus Michx.]

Dalea enneandra Nutt.

[=Parosela enneandra (Nutt.) Britton]

Dalea multiflora (Nutt.) Shinners

[=Petalostemon multiflorus Nutt.]

Dalea purpurea Vent.

[=Petalostemon purpureus (Vent.) Rydb.]

Dalea sp.

[=Petalostemon gracilis Nutt., misapplied]

Desmanthus illinoensis (Michx.) MacMill. ex

B.L. Rob. \& Fernald

Desmodium cuspidatum (Muhl. ex Willd.) DC.

[=Desmodium grandiflorum DC.]

Desmodium sessilifolium (Torr.) Torr. \&

A. Gray

Glycyrrhiza lepidota Pursh

Gymnoclados dioicus (L.) K. Koch

Hoffmannseggia glauca (Ortega) Eifert

[=Hoffmannseggia falcaria Cav.]

Indigofera miniata Ortega

[=Indigofera leptosepala Nutt. ex Torr. \&

A. Gray]

Lathyrus pusillus Elliott

Lotus unifoliolatus (Hook.) Benth.

[=Hosackia americana (Nutt.) Piper] lead-plant, devil's shoe-string

river-locust, false indigo

tall astragalus

ground plum

low astragalus

Annual astragalus

blue false indigo

false indigo

redbud, Judas tree

partridge pea

golden parosela

white prairie clover

slender parosela

round-headed prairie clover

purple prairie-clover

slender white prairie clover

Illinois desmanthus

pointed-leaved tick trefoil, sticktight

sessile-leaved tick-trefoil

wild liquorice

Kentucky coffee-tree

blue-weed

western indigo plant

low wild pea

prairie bird's foot, trefoil sandy soils, near

rivers; summer

creeks; summer

streams; summer

creeks; summer

creeks, pastures;

spring

prairies; spring

prairies; spring

mountainsides;

spring

mountainsides;

spring

creeks, mountain

ravines; pre-vernal

fields, pastures;

summer

hillsides; summer

near rivers, sandy

soils; summer

rivers; summer

prairies; summer

prairies; summer

prairies; summer

rivers; summer

prairies; summer

mountains; summer

dry sands,

roadsides; summer

ravines; spring

prairies; spring

prairies; summer to

fall

rivers; spring

prairies; summer 
Medicago sativa L.

Melilotus officinalis (L.) Lam.

Mimosa microphylla Dryand.

[=Schrankia angustata Torr. \& A. Gray,

Schrankia uncinata Willd.]

Neptunia lutea (Leavenworth) Benth.

Pediomelum cuspidatum (Pursh) Rydb.

[=Psoralea cuspidata Pursh]

Pomaria jamesii (Torr. \& A. Gray) Walp.

[=Hoffmannseggia jamesii Torr. \& A. Gray]

Prosopis glandulosa Torr. var. glandulosa

[=Prosopis juliflora (Sw.) DC. var. glandulosa

(Torr.) Cockerell]

Psoralidium tenuiflorum (Pursh) Rydb.

[=Psoralea tenuiflora Pursh]

Robinia pseudoacacia L.

Vicia caroliniana Walter

\section{Liliaceae [incl. Amaryllidaceae]}

Allium canadense L. var. mobilense (Regel) wild onion

Ownbey [=Allium mutabile Michx.]

Allium drummondii Regel

[=Allium nuttallii S. Watson]

Allium textile A. Nelson \& J.F. Macbr.

[=Allium reticulatum $\mathrm{G}$. Don]

Androstephium coeruleum (Scheele) Greene

Camassia scilloides (Raf.) Cory

[=Camassia esculenta (Raf.) Cory]

Cooperia drummondii Herb.

Nothoscordum bivalve (L.) Britton

Polygonatum biflorum (Walter) Elliott alfalfa

yellow meliot, sweet clover

sensitive brier

neptunia

large-bracted psoralea

James's hoffmannseggia

prairie mesquite

few-flowered psoralea

black or yellow locust

pale vetch

Nuttall's wild onion

wild onion

androstephium

hyacinth, eastern camas

prairie lily

yellow false garlic

great Solomon's seal escaped cultivation, fields, roadsides; spring, summer roadsides; abundant; summer roadside ditches; common; summer

mountain ravines; summer prairies; spring

prairies; early summer prairies; common; late spring

prairies; spring to fall

low waste places, cultivated; summer prairies; spring

damp soils, roadsides; spring roadsides; spring

mountain ravines; late spring prairies, rare; early spring mountain ravines

mountain ravines; spring pastures, fields; spring, fall damp shady places; summer

roadsides; spring prairies; spring prairies; summer
Lewis's wild flax

large-flowered yellow flax
Linum rigidum Pursh

Linum sulcatum Riddell

\section{Linaceae}




\section{Loasaceae}

Mentzelia decapetala (Pursh ex Sims)

Urb. \& Gilg ex Gilg

Mentzelia oligosperma Nutt. ex Sims

\section{Lythraceae}

Ammannia coccinea Rottb.

\section{Malvaceae \\ Callirhoe involucrata (Torr. \& A. Gray) A. Gray \\ Callirhoe papaver (Cav.) A. Gray \\ Sphaeralcea coccinea (Nutt.) Rydb. \\ [=Malvastrum coccineum (Nutt.) A. Gray] \\ Martyniaceae \\ Proboscidea louisianica (Mill.) Thell. \\ [=Martynia louisiana Mill.]}

\section{Menispermaceae}

Cocculus carolinus (L.) DC.

\section{Moraceae [Urticaceae]}

Morus rubra L.

\section{Nyctaginaceae}

Mirabilis albida (Walter) Heimerl

[=Oxybaphus albidus (Walter) Sweet]

Mirabilis hirsuta (Pursh) MacMill.

[=Oxybaphus hirsutus (Pursh) Sweet]

Mirabilis linearis (Pursh) Heimerl

[=Oxybaphus linearis (Pursh) B.L. Rob.]

Mirabilis nyctaginea (Michx.) MacMill.

[=Oxybaphus nyctagineus (Michx.) Sweet]

\section{Oleaceae}

Fraxinus americana L.

Fraxinus pennsylvanica Marshall

\section{Onagraceae}

Ludwigia peploides (Kunth) P.H. Raven

[=Jussiaea repens L.] stick-leaf

stick-leaf, few seeded

mentzelia

long-leaved ammannia

purple poppy mallow

larger purple poppy mallow

red false-mallow

unicorn plant

Carolina moonseed

red mulberry; wild mulberry

white oxybaphus

hairy oxybaphus

oxybaphus

petioled wild four-o'clock

white ash

red ash

creeping primrose-willow creeks; spring

sand, near rivers;

summer

rivers; summer

mountain ravines;

summer

roadside ditches;

common; spring

creeks; spring,

summer

roadsides; common; spring

cultivated soils;

summer

streams; summer

roadsides, dry sand;

summer

dry soils, roadsides;

summer

prairies; summer

creeks; spring

creeks; spring,

summer

mountain ravines;

spring

ponds; summer 
Oenothera cinerea (Wooton \& Standl.) W.L. wooly gaura creeks; summer

Wagner \& Hoch [=Gaura villosa Torr.]

Oenothera curtiflora W.L. Wagner \& Hoch gaura

[=Gaura parviflora Douglas ex Lehm.]

Oenothera glaucifolia W.L. Wagner \& Hoch flax-leaved stenosiphon

[=Stenosiphon linifolius (Nutt. ex E. James)

Heynh.]

Oenothera grandis (Britton) Smyth

[=Oenothera laciniata Hill var. grandiflora

(S. Watson) B.L. Rob.]

Oenothera hartwegii Benth.

Oenothera laciniata Hill

Oenothera macrocarpa Nutt.

[=Oenothera missouriensis Sims]

Oenothera rhombipetala Nutt. ex Torr. \&

A. Gray

Oenothera serrulata Nutt.

Oenothera sinuosa W.L. Wagner \& Hoch

[=Gaura sinuata Nutt. ex Ser.]

Oenothera speciosa Nutt.

Oenothera suffrutescens (Ser.) W.L. Wagner

$\&$ Hoch [=Gaura coccinea Nutt. ex Pursh]

Oenothera triloba Nutt.

Oenothera sp.

[=Gaura biennis L., misapplied]

Oenothera sp.

[=Oenothera humifusa Nutt., misapplied]

Oenothera sp.

[=Oenothera oakesiana (A. Gray) J.W.

Robbins ex S. Watson \& J.M. Coult., misapplied]

\section{Oxalidaceae}

Oxalis corniculata L.

Oxalis stricta L.

Oxalis violacea $\mathrm{L}$. dry sandy soils;

summer

sandy soils near

rivers; summer

evening-primrose

hillsides; spring

evening-primrose

evening-primrose

Missouri evening-primrose

evening-primrose

tooth-leaved primrose

wavy-leaved gaura

showy evening-primrose

scarlet gaura

three-lobed evening-primrose

biennial gaura

evening-primrose

evening-primrose plains; summer

sand, near rivers;

summer

hillsides; summer

near rivers; summer

pastures, roadsides;

summer

hillsides; summer

prairies; spring

roadside ditches, mountainsides;

spring

rivers; summer

rivers; summer

near rivers; summer

sandy soils, near

rivers; summer damp soils,

mountainsides;

spring

damp soils, mountainsides;

spring

violet wood-sorrel damp soils, creeks, mountainsides;

spring 


\section{Papaveraceae}

Argemone albiflora Hornem.

[=Argemone alba Lestib. f.]

Argemone polyanthemos (Fedde) G.B.

Ownbey [=Argemone intermedia Sweet]

\section{Phrymaceae}

Phryma leptostachya L.

\section{Phytolaccaceae}

Phytolacca americana L.

\section{Plantaginaceae \\ Plantago aristata Michx. \\ Plantago patagonica Jacq. \\ [=Plantago purshii Roem. \& Schult.] \\ Plantago rhodosperma Decne. \\ Plantago virginica $\mathrm{L}$. \\ Polemoniaceae \\ Ipomopsis rubra (L.) Wherry \\ [=Gilia rubra (L.) A. Heller] \\ Phlox pilosa $\mathrm{L}$.}

\section{Polygalaceae}

Polygala alba Nutt.

\section{Polygonaceae}

Eriogonum annuum Nutt.

Eriogonum longifolium Nutt.

Polygonum aviculare $\mathrm{L}$.

Polygonum hydropiper L.

Polygonum lapathifolium L.

Polygonum pensylvanicum L.

Polygonum punctatum Elliott

[=Polygonum acre Kunth]

Polygonum ramosissimum Michx.

Polygonum tenue Michx.

Rumex altissimus Alph. Wood

Rumex crispus L.

[incl. Rumex elongatus Guss.] white prickly poppy

prickly poppy

lop-seed

pokeweed

ribwort

Pursh's plantain

red-seeded plantain

dwarf plantain

red gilia

phlox

white milkwort

annual gray-weed

long-leaved gray-weed

joint-weed, pink-weed

common smart-weed

dock-leaved joint-weed

showy joint-weed

water smart-weed, dotted

water pepper

bushy joint-weed

slender joint-weed

tall dock

dock, curly dock mountains; summer

creek banks;

summer

roadsides; spring,

summer

roadsides; summer

prairies; common;

spring

pastures, roadsides;

spring

rivers; early spring

creeks; spring

mountainsides;

summer

prairie roadsides;

spring, summer

prairies; summer

prairies; summer

rivers; summer, fall

near dwellings;

summer

lakes; late summer

ravines; summer

streams; summer

mountain ravines;

summer, fall

rivers; summer

mountains; summer

roadsides; summer

damp soils,

mountains; spring,

summer 
Portulacaceae [incl. Caryophyllaceae, in part]

Claytonia virginica $\mathrm{L}$.

spring beauty

androsace

water pimpernel, brookweed

[=Samolus floribundus Kunth]

\section{Ranunculaceae}

Anemone berlandieri Pritz. [=Anemone decapetala Ard.]

Anemone caroliniana Walter

Clematis pitcheri Torr. \& A. Gray

Delphinium carolinianum Walter ssp. virescens (Nutt.) R.E. Brooks [=Delphinium penardii Huth]

Myosurus minimus L.

\section{Rhamnaceae}

Ceanothus americanus $\mathrm{L}$.

New Jersey tea

ten-petaled anemone

Carolina anemone

virgin's bower, leather-flower

larkspur

mouse tail

pastures; spring

pastures; common; March, April creeks, mountain ravines; spring mountains, roadsides; spring

streams, fields; early spring

mountains, streams; spring

\section{Rosaceae}

Crataegus crus-galli L.

Crataegus viridis $\mathrm{L}$.

Geum canadense Jacq.

Prunus americana Marshall

Prunus angustifolia Marshall

Rubus argutus Link

Rubus sp.

[=Rubus baileyanus Britton, misapplied]

\section{Rubiaceae}

Cephalanthus occidentalis L.

Diodia teres Walter

Galium aparine L. cock-spur haw, red raw

southern thorn

white avena

wild yellow or red plum

Chickasaw plum

bramble

Bailey's blackberry

rough button-weed

cleavers button-bush rivers; spring

streams; early

spring

mountain ravines;

summer

rivers; spring roadside ditches, near rivers; spring rivers; spring mountainsides; spring

streams, mountains; late spring rivers; summer mountains, streams; spring 
Galium pilosum Aiton

Houstonia pusilla Schoepf

[=Houstonia minima Beck]

Stenaria nigricans (Lam.) Terrell

[=Houstonia angustifolia Michx.]

\section{Rutaceae}

Ptelea trifoliata L.

\section{Salicaceae}

Populus deltoides W. Bartram ex Marshall

Salix nigra Marshall

\section{Sapotaceae}

Sideroxylon lanuginosum Michx.

[=Bumelia lanuginosa (Michx.) Pers.]

\section{Smilacaceae [Liliaceae]}

Smilax bona-nox L.

Smilax herbacea L.

Smilax rotundifolia L.

\section{Santalaceae}

Comandra umbellata (L.) Nutt. ssp. pallida

(A. DC.) Piehl

[=Comandra pallida A. DC.]

\section{Sapindaceae}

Sapindus saponaria L. var drummondii (Hook. \& Arn.) L.D. Benson

[=Sapindus drummondii Hook. \& Arn.]

\section{Scrophulariaceae}

Castilleja purpurea (Nutt.) G. Don var. lindheimeri (A. Gray) Shinners

[=Castilleja lindheimeri A. Gray]

Castilleja sessiliflora Pursh

Collinsia violacea Nutt.

Nuttallanthus canadensis (L.) D.A. Sutton [=Linaria canadensis (L.) Chaz.]

hairy bedstraw

bluets

narrow-leaved houstonia

tree-leaved hop-tree

mountainsides;

spring

cottonwood, necklace poplar

black willow

creeks; pre-vernal

damp soils,

streams; spring

chittim-wood, wooly buckthorn

mountainsides;

summer

spiny-leaved greenbrier

carrion flower

creeks; spring

mountain ravines;

summer

common greenbrier,

horse-brier

creeks; spring

bastard toad-flax

dry sandy soils, near rivers; spring

wild China-tree, Drummond's creeks; spring soapberry

Indian paint brush

downy painted-cup

violet or narrow-leaved

collinsia

linaria mountains, pastures; spring

mountainsides;

summer

mountainsides;

spring

mountains; spring 
Penstemon cobaea Nutt.

Penstemon tubaeflorus Nutt.

Penstemon sp.

[=Penstemon acuminatus Douglas

ex Lindl., misapplied]

Veronica agrestis L.

Veronica peregrina L.

\section{Solanaceae}

Chamaesaracha sp.

[=Chamaesaracha sordida (Dunal) A. Gray, misapplied]

Datura stramonium L.

Physalis cinerascens (Dunal) Hitchc. [=Physalis viscosa L., misapplied]

Physalis longifolia Nutt.var. Iongifolia

Physalis longifolia Nutt. var. subglabrata

\{Mack. \& Bush) Cronq.

[=Physalis subglabrata Mack. \& Bush]

Physalis mollis Nutt.

Quincula lobata (Torr.) Raf.

[=Physalis lobata Torr.]

Solanum carolinense L.

Solanum elaeagnifolium Cav.

Solanum rostratum Dunal

Solanum sp.

[=Solanum nigrum L., misapplied]

\section{Tamaricaceae}

Tamarix sp.

[=Tamarix gallica L., misapplied]

\section{Typhaceae}

Typha latifolia L.

\section{Ulmaceae [Urticaceae]}

Celtis laevigata Willd.

Celtis occidentalis L.

Ulmus americana L. beard-tongue

funnel-shaped beard-tongue

sharp-leaved beard-tongue

field speedwell

neckweed purslane, I

speedwell

hairy chamaesaracha

jimson weed

ground-cherry

smooth ground-cherry

smooth ground-cherry

velvety ground-cherry

purple-flowered ground-cherry

horse nettle

horse nettle

buffalo bur

nightshade

tamarish

broad-leaved cat-tail

southern hackberry

rough-leaved hackberry

white, American, or water elm roadsides; summer

ponds; summer

prairies; late spring

creeks; summer

hillsides; summer

fields, pastures;

spring

creeks; early spring

roadsides; summer

creeks; spring

creeks; spring

sandy soils, near

rivers; summer

roadsides; summer

roadsides, prairies;

spring, early

summer

prairies; common;

spring, summer

pastures, roadsides;

common; spring,

summer

abundant; summer, fall

dry sandy soils;

summer

damp sandy soils;

summer

creeks; spring mountains, streams; early spring

creeks; early spring 
Ulmus rubra Muhl.

[=Ulmus fulva Michx.]

\section{Umbelliferae}

Chaerophyllum tatinturieri Hook.

Daucus pusillus Michx.

Lomatium foeniculaceum (Nutt.) J.M. Coult.

\& Rose ssp. daucifolium (Torr. \& A. Gray)

W.L. Theobald [=Lomatium daucifolium

(Torr. \& A. Gray) J.M. Coult. \& Rose]

Ptilimnium nuttallii (DC.) Britton

Sanicula canadensis L.

Spermolepis echinata (Nutt. ex DC.) A. Heller

Spermolepis inermis (Nutt. ex DC.) Mathias

$\&$ Constance [=Spermolepis patens (Nutt.

ex DC.) B.L. Rob.]

\section{Valerianaceae}

Valerianella radiata (L.) Dufr.

\section{Verbenaceae}

Glandularia bipinnatifida (Nutt.) Nutt.

[=Verbena bipinnatifida Nutt.]

Glandularia canadensis (L.) Nutt.

[=Verbena canadensis (L.) Britton]

Glandularia pumila (Rydb.) Umber

[=Verbena pumila Rydb.]

Phyla cuneifolia (Torr.) Greene

[=Lippia cuneifolia (Torr.) Steud.]

Phyla lanceolata (Michx.) Greene

[=Lippia lanceolata Michx.]

Phyla nodiflora (L.) Greene

[=Lippia nodiflora (L.) Michx.]

Verbena bracteata Cav. ex Lag. \& Rodr.

[=Verbena bracteosa Michx.]

Verbena stricta Vent. slippery or red elm

Teinturier's chervil

American carrot

carrot-leaved parsley

Nuttall's mock bishop-weed

short-styled snake-root

bristly-fruited spermolepis

spreading spermolepis

beaked corn salad

small-flowered verbena

large-flowered verbena

dwarf verbena

wedge-leaved fog-fruit

fog-fruit

spatulate-leaved fog-fruit

large-bracted verbena

hoary vervain

pansy or heart's ease mountain ravines;

spring

roadsides; common; spring

fields, pastures;

spring

rivers; summer low places near mountains; summer mountainsides; summer mountainsides; spring rivers; spring

creeks; spring

creeks, pastures; early spring, summer sandy soils, near rivers; spring roadsides; summer

hillsides; summer

creeks; summer

streams; summer

prairies; summer

mountainsides; summer

streams, pastures; early spring 
Viola sororia Willd.

[=Viola papilionacea Pursh]

\section{Vitaceae}

Ampelopsis cordata Michx. [=Cissus ampelopsis Pers.]

Cissus trifoliata (L.) L.

[=Cissus incisa Des Moulins, misapplied]

Parthenocissus quinquefolia (L.) Planch.

Vitis cinerea (Engelm.) Engelm. ex Millard

Vitis vulpina $\mathrm{L}$.

[incl. Vitis cordifolia Michx.]

\section{Zygophyllaceae}

Kallstroemia parviflora J.B.S. Norton [=Kallstroemia maxima (L.) Hook. \& Arn., misapplied]

Tribulus terrestris L. violet

damp sandy soils; early spring

simple-leaved cissus

rock-grape

Virginia creeper, five-leaved ivy ashy or downy grape frost-grape, sweet scented grape

greater caltrop

caltrop rivers; spring

mountains; summer

creeks; summer

streams; spring creeks, rivers; spring

rivers; summer

roadsides; common; summer 


\section{APPENDIX B}

Tabular List of the Families, Kiowa County, OK

[This table includes taxa as they were in the original thesis.]

\begin{tabular}{|c|c|c|c|}
\hline Divisions, Orders, Families, Etc. & Genera & Species & Varieties \\
\hline \multicolumn{4}{|l|}{ PTERIDOPHYTA } \\
\hline \multicolumn{4}{|l|}{ Filicales } \\
\hline Polypodiaceae & 4 & 5 & \\
\hline Marsileaceae & 1 & 1 & \\
\hline \multicolumn{4}{|l|}{ SPERMATOPHYTA } \\
\hline \multicolumn{4}{|l|}{ Gymnospermae } \\
\hline \multicolumn{4}{|l|}{ Coniferales } \\
\hline Pinaceae & 1 & 1 & \\
\hline \multicolumn{4}{|l|}{ Angiospermae } \\
\hline \multicolumn{4}{|l|}{ MONOCOTOLEDONEAE } \\
\hline \multicolumn{4}{|l|}{ Pandales } \\
\hline Typhaceae & 1 & 1 & \\
\hline \multicolumn{4}{|l|}{ Graminales } \\
\hline Gramineae & 31 & 58 & 2 \\
\hline Cyperaceae & 6 & 11 & \\
\hline \multicolumn{4}{|l|}{ Xyridales } \\
\hline Commelinaceae & 2 & 6 & \\
\hline \multicolumn{4}{|l|}{ Liliales } \\
\hline Juncaceae & 1 & 6 & \\
\hline Liliaceae & 7 & 11 & \\
\hline Amaryllidaceae & 1 & 1 & \\
\hline Iridaceae & 1 & 3 & \\
\hline \multicolumn{4}{|l|}{ DICOTYLEDONEAE } \\
\hline \multicolumn{4}{|l|}{ Salicales } \\
\hline Salicaceae & 2 & 2 & \\
\hline \multicolumn{4}{|l|}{ Juglandales } \\
\hline Juglandaceae & 2 & 3 & \\
\hline \multicolumn{4}{|l|}{ Fagales } \\
\hline Fagaceae & 1 & 6 & \\
\hline \multicolumn{4}{|l|}{ Urticales } \\
\hline Urticaceae & 4 & 5 & 1 \\
\hline \multicolumn{4}{|l|}{ Santalales } \\
\hline Santalaceae & 1 & 1 & \\
\hline \multicolumn{4}{|l|}{ Polygonales } \\
\hline Polygonaceae & 3 & 12 & \\
\hline Chenopodiales & & & \\
\hline
\end{tabular}

Lottie Opal Baldock 


\begin{tabular}{|l|c|c|c|}
\hline Chenopodiaceae & 4 & 5 & 1 \\
\hline Amaranthaceae & 3 & 5 & \\
\hline Phytolaccaceae & 1 & 1 & \\
\hline Nyctaginaceae & 1 & 4 & \\
\hline Illecebraceae & 1 & 1 & \\
\hline Aizoaceae & 1 & 1 & \\
\hline Caryophyllales & & & \\
\hline Caryophyllaceae & 3 & 4 & \\
\hline Portulacaceae & 1 & 1 & \\
\hline Ranunculales & & & \\
\hline Ranunculaceae & 4 & 5 & \\
\hline Menispermaceae & 1 & 1 & \\
\hline Papavervales & & & \\
\hline Papaveraceae & 1 & 2 & \\
\hline Fumariaceae & 2 & 2 & \\
\hline Cruciferae & 8 & 16 & \\
\hline Capparidaceae & 2 & 2 & \\
\hline Rosales & & & \\
\hline Crassulaceae & 1 & 1 & \\
\hline Saxifragaceae & 1 & 1 & \\
\hline Rosaceae & 4 & 7 & \\
\hline Leguminosae & 25 & 42 & 20 \\
\hline Geraniales & & & \\
\hline Linaceae & 1 & 3 & \\
\hline Oxalidaceae & 1 & 3 & \\
\hline Geraniaceae & 1 & 1 & \\
\hline Zygophyllaceae & 1 & 2 & \\
\hline Rutaceae & 1 & 1 & \\
\hline Polygalaceae & 1 & 1 & \\
\hline Euphorbiaceae & 5 & 16 & 1 \\
\hline Sapindales & & & \\
\hline Anacardiaceae & 2 & 4 & \\
\hline Sapindaceae & 1 & 1 & \\
\hline Rhamnales & 1 & 1 & \\
\hline Rhamnaceae & 3 & 6 & \\
\hline Vitaceae & & & \\
\hline Malvales & 2 & 3 & \\
\hline Malvaceae & & & \\
\hline Tamaricales & 1 & 1 & \\
\hline Tamaricaceae & & & \\
\hline Violales & & 2 & \\
\hline Violaceae & & \\
\hline Loasaceae & & \\
\hline & & & \\
\hline
\end{tabular}

\section{Opuntiales}




\begin{tabular}{|c|c|c|c|}
\hline Cactaceae & 2 & 2 & \\
\hline \multicolumn{4}{|l|}{ Myrtales } \\
\hline Lythraceae & 1 & 1 & \\
\hline Onagraceae & 4 & 17 & 1 \\
\hline \multicolumn{4}{|l|}{ Umbellales } \\
\hline Umbelliferae & 6 & 7 & \\
\hline Cornaceae & 1 & 2 & \\
\hline \multicolumn{4}{|l|}{ Primulales } \\
\hline Primulaceae & 2 & 2 & \\
\hline \multicolumn{4}{|l|}{ Ebenales } \\
\hline Sapotaceae & 1 & 1 & \\
\hline Ebenaceae & 1 & 1 & \\
\hline \multicolumn{4}{|l|}{ Gentianales } \\
\hline Oleaceae & 1 & 2 & \\
\hline Gentianaceae & 2 & 3 & \\
\hline Apocynaceae & 2 & 2 & \\
\hline Asclepiadaceae & 4 & 11 & \\
\hline \multicolumn{4}{|l|}{ Polemoniales } \\
\hline Convolvulaceae & 3 & 6 & \\
\hline Polemoniaceae & 2 & 2 & \\
\hline Hydrophyllaceae & 1 & 2 & \\
\hline Boraginaceae & 5 & 6 & 1 \\
\hline Verbenaceae & 2 & 8 & \\
\hline Labiatae & 6 & 11 & 1 \\
\hline Solanaceae & 4 & 12 & \\
\hline Scrophulariaceae & 5 & 9 & \\
\hline Martyniaceae & 1 & 1 & \\
\hline Acanthaceae & 1 & 2 & \\
\hline Phrymaceae & 1 & 1 & \\
\hline \multicolumn{4}{|l|}{ Plantaginales } \\
\hline Plantaginaceae & 1 & 4 & \\
\hline \multicolumn{4}{|l|}{ Rubiales } \\
\hline Rubiaceae & 4 & 6 & \\
\hline Caprifoliaceae & 4 & 2 & \\
\hline Valerianaceae & 1 & 1 & \\
\hline \multicolumn{4}{|l|}{ Campanulales } \\
\hline Curcurbitaceae & 1 & 1 & \\
\hline Campanulaceae & 1 & 2 & \\
\hline Lobeliaceae & 1 & 2 & \\
\hline Compositae & 42 & 86 & 2 \\
\hline
\end{tabular}




\section{ADDENDA}

[Nomenclature has been updated according to the PLANTS database (http://plants.usda.gov/plants).]

The following plants were counted in the tabular list but are not given in the list of species:

Artemisia filifolia

Desmodium obtusum

Draba reptans [Draba caroliniana]

Eleocharis obtusa

Gaillarida suavis [Gaillardia trinervata]

Juncus tenuis

Physalis virginiana

Rudbeckia hirta

Scutellaria parvula

Symphyotrichum oblongifolium [Aster oblongifollius]

Vicia minutiflora [Vicia micrantha]

Xanthisma texanum

The following plants listed in the Stevens' collection were not found by the author:

Artemisia ludoviciana spp. mexicana [Artemisia mexicana]

Beta vulgaris

Bouteloua rigidiseta [Bouteloua texana]

Carex gravida

Distichlis spicata

Dyssodiopsis tagetoides [Dyssodia tagetoides]

Eleocharis rostellata

Muhlenbergia arenicola

Palafoxia sphacelata

Samolus ebracteatus 